\title{
Food insecurity is associated with suboptimal sleep quality, but not sleep duration, among low-income Head Start children of pre-school age
}

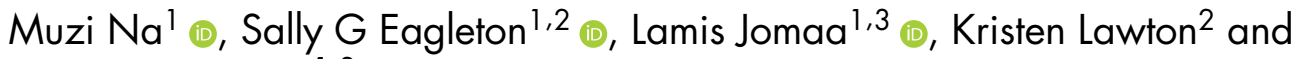 \\ Jennifer S Savage ${ }^{1,2, *}$ \\ 'Department of Nutritional Sciences, Penn State College of Health and Human Development, 108C Chandlee \\ Laboratory, University Park, PA 16802, USA: ${ }^{2}$ Center for Childhood Obesity Research, Penn State College of Health \\ and Human Development, University Park, PA, USA: ${ }^{3}$ Department of Nutrition and Food Sciences, Faculty of \\ Agricultural and Food Sciences, American University of Beirut, Beirut, Lebanon
}

Submitted 8 January 2019: Final revision received 2 July 2019: Accepted 24 July 2019: First published online 28 November 2019

\begin{abstract}
Objective: To examine the association between food insecurity and child sleep outcomes and to investigate whether parent psychosocial factors mediate such associations.

Design: Cross-sectional study. Usual wake time and bedtime, bedtime routine and sleep quality were reported by parents using the adapted Brief Infant Sleep Questionnaire. Food insecurity was assessed using the eighteen-item US Department of Agriculture Household Food Security Module. Parent psychosocial factors, including perceived stress, parenting self-efficacy and depressive symptomology, were assessed using validated scales. Multivariable logistic regression models were performed to determine the association between food insecurity and sleep outcomes controlling for potential confounders. Mediation analyses and Sobel tests were applied to test the mediating effect of psychosocial factors. Setting: Head Start pre-school classrooms in four regions across central Pennsylvania, USA.

Participants: Low-income children of pre-school age ( $n$ 362) and their caregivers. Results: Prevalence of household, adult and child food insecurity was $37 \cdot 3,31 \cdot 8$ and $17.7 \%$, respectively. Food security status at any level was not associated with child sleep duration or bedtime routine. Child food insecurity, but not household or adult food insecurity, was associated with 2.25 times increased odds (95\% CI 1.11, 4.55) of poor child sleep quality in the adjusted model. Perceived stress, selfefficacy and depressive symptomology mediated less than $2 \%$ of the observed effect (all Sobel test $P>0 \cdot 6$ ).

Conclusion: Food insecurity, particularly at the child level, is a potential modifiable risk factor for reducing sleep-related health disparities in early childhood. Future studies are needed to explore the plausible mechanisms underlying the associations between food insecurity and adverse child sleep outcomes.
\end{abstract}

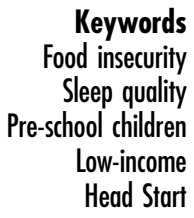

s.

Sleep quality

Head Start
Data from large-scale epidemiological surveys has revealed that about one in four young children in developed countries experiences a range of sleep problems ${ }^{(1,2)}$. Poor child sleep, characterized by insufficient sleep duration or suboptimal sleep quality, is linked to poor academic and educational outcomes ${ }^{(3,4)}$. Cumulative evidence summarized by recent systematic reviews and meta-analyses supports a consistent association between short sleep duration in childhood and obesity risk later in life ${ }^{(5-7)}$. Further, among toddlers and children of pre-school age, sleep problems are associated with increased risk of emotional and behavioural problems in the school-age years ${ }^{(8,9)}$ and early adulthood $^{(10)}$. Understanding the contextual factors associated with poor child sleep is critical to identify, target and intervene at an early age to achieve healthy development and better health outcomes across the lifespan.

Food insecurity, defined as a limited or uncertain availability of nutritionally adequate and safe foods or the 
limited ability to acquire food in socially acceptable ways $^{(11)}$, has been proposed as a stressor that influences health-related behaviours, such as sleep and adverse mental health indicators. Several large population-based surveys indicate that food-insecure adults are more likely to report shorter sleep duration ${ }^{(12-15)}$, poorer sleep quality ${ }^{(14)}$, longer sleep latency and more sleep complaints ${ }^{(12)}$ compared with food-secure adults. However, this research has been limited primarily to adults. According to the US Department of Agriculture, nearly 6.5 million children under the age of 18 years lived in food-insecure households in 2017 and the prevalence of food-insecure households with children under the age of 6 years was $16.4 \%$, a number that was higher than the national average of $11.8 \%{ }^{(16)}$. Although the exact mechanism is still unclear, poor sleep could be a potential mechanism through which food insecurity may affect various chronic health conditions that may emerge in childhood, including increased cardiometabolic risk $^{(17)}$, coexisting obesity ${ }^{(18)}$ and behavioural and emotional problems ${ }^{(19)}$.

Although research efforts have been directed at better understanding the health implications of food insecurity, little is known about the sleep quantity and quality of young children in relation to their food security status. The primary aim of the present study was to examine the association between food security and sleep outcomes in a sample of children of pre-school age from low-income families enrolled in Head Start. Food insecurity is commonly associated with parent stress, anxiety and depressive symptoms ${ }^{(20)}$, which in turn, may influence parenting $^{(21)}$ and child sleep behaviours ${ }^{(22)}$. Thus, the secondary aim of the study was to explore whether parent psychosocial factors, namely perceived stress, parenting self-efficacy and depressive symptomology, mediated any observed associations between food security status and child sleep outcomes.

\section{Methods}

\section{Study design and participants}

The current study utilized data from a needs assessment survey designed to characterize the home environments of families among a disadvantaged population of caregivers with young children. The survey was distributed to over 1000 caregivers of children enrolled in Head Start pre-school classrooms within four rural counties in central Pennsylvania, USA. Head Start teachers distributed the survey, which included informed consent and instructions for completing and returning the survey packet using a prepaid envelope. Caregivers were mailed a \$US 25 gift card for participation. Data collection spanned May 2017-May 2018. The response rate was $29.6 \%$ (379 surveys returned of the 1279 distributed). Given that the majority of caregiver respondents were parents (90\%), we refer to caregivers as 'parents' throughout. Parents were instructed to complete the survey for their oldest child enrolled in Head Start. Nine surveys were excluded because a parent had completed a survey for more than one child. Four participants were ineligible based on the child's date of birth (e.g. age outside the range of Head Start pre-school eligibility) and an additional four were removed due to missing data, resulting in a final study sample of 362 . The study was approved by the Office for Research Protections at The Pennsylvania State University, University Park, PA, USA.

\section{Child sleep}

Child sleep duration was calculated based on parent reports of their child's typical bedtime and wake time in hours and minutes. Two items from an expanded version of the Brief Infant Sleep Questionnaire (BISQ) ${ }^{(23)}$ were used to measure bedtime routine and sleep quality. Bedtime routine was assessed with parent reports of how often ( $1=$ 'never' to $5=$ 'every night') their child has the same bedtime routine in a typical $7 \mathrm{~d}$ week. Sleep quality was assessed with parent ratings of how well their child usually sleeps at night ( $1=$ 'very poorly' to $6=$ 'very well'). Sleep quality was grouped as 'poor' (if response was 1-5) and 'very well' (if response was 6). The BISQ has been validated against actigraphy and sleep $\operatorname{logs}^{(24)}$ and used in previous studies with pre-school children ${ }^{(25,26)}$.

\section{Food security status}

The eighteen-item US Department of Agriculture Household Food Security Module was used to measure household, adult and child food security status ${ }^{(11)}$. Households and adults were independently classified as 'food insecure' if participants responded affirmatively to three or more items and 'food secure' if two or fewer items were affirmative on the respective full eighteen-item scale and the ten-item adult food security subscale. Child food security status was assessed using the eight childreferenced questions and children were considered 'food insecure' if participants responded affirmatively to two or more items ${ }^{(27)}$.

\section{Parent psychosocial factors}

Parent perceived stress levels were assessed using the tenitem Perceived Stress Scale (PSS-10) ${ }^{(28)}$, a widely used and validated measure of the degree to which respondents believe their life to be unpredictable, uncontrollable and overwhelming ${ }^{(29)}$. Response options for the ten items ( $\alpha=0.85)$ ranged from $1=$ 'never' to $5=$ 'very often'. A standardized PSS-10 $Z$-score ( mean $=0, \mathrm{SD}=1$ ) was calculated, with higher scores indicating higher levels of stress over the past month.

The five-item version of the Parenting Sense of Competency self-efficacy subscale (PSOC-5) ${ }^{(30)}$ was used to assess general parenting confidence. The PSOC-5 was validated in a large, non-clinical sample of parents ${ }^{(30)}$ and has been reliably used among mothers with children 
of pre-school age ${ }^{(31)}$. Response options for the five items $(\alpha=0.79)$ ranged from $1=$ 'strongly agree' to $6=$ 'strongly disagree'. All items were reverse coded, a total PSOC-5 score was calculated and then standardized such that higher scores reflect greater perceived parenting selfefficacy.

The Center for Epidemiological Studies Depression Scale-10 (CESD-10) ${ }^{(32)}$, a well-validated measure with acceptable test-retest and predictive validity, was used to assess parent depressive symptomology. Response options for the ten items $(\alpha=0.82)$ ranged from $0=$ 'rarely or not at all' to $3=$ 'most days.' A total CESD-10 score was calculated and then standardized, with higher scores indicating higher levels of depressed mood during the past week.

\section{Covariates}

Key parent demographic characteristics were considered as covariates to adjust for potential confounding effects, including child age and sex, parent age, education, marital status, employment status, number of children in the household, number of people supported by household income, participation in assistance programmes (e.g. Special Supplemental Nutrition Program for Women, Infants, and Children (WIC); Supplemental Nutrition Assistance Program (SNAP)), household income and region. The analytic sample was constrained to participants who had complete data on food insecurity, sleep outcomes and adjusting covariates. Household income was missing in seventy-four of 362 households (20.4\%). Missing income was imputed based on WIC and SNAP status, parent education, marital status and employment using PROC MI in SAS and imputed income was adjusted in sensitivity analysis.

Given previous research showing an association between factors within the home environment and child sleep ${ }^{(33,34)}$, household chaos and family functioning were also considered as covariates. Household chaos was assessed using the Confusion, Hubbub, and Order Scale $(\mathrm{CHAOS})^{(35)}$, a measure of structural disorganization in the home marked by high noise levels, crowding, and lack of predictability and family routines. The concurrent validity of the scale was demonstrated against direct observational measures in the home and has acceptable test-retest reliability ${ }^{(35)}$. CHAOS has been used with diverse samples of parents with young children ${ }^{(36,37)}$. The item 'The telephone takes up a lot of our time at home' was replaced with 'There is usually a television turned on somewhere in our home $^{(38)}$. Response options for the fifteen items $(\alpha=0.86)$ ranged from $1=$ 'definitely untrue' to $5=$ 'definitely true'. A mean score was calculated with higher scores indicating greater chaos. Family functioning was assessed with the general family functioning subscale from the Family Assessment Device (FAD) ${ }^{(39)}$. The FAD is widely used and has been shown to have acceptable test-retest reliability and concurrent and discriminant validity ${ }^{(40)}$. Response options for the twelve items $(\alpha=0.91)$ ranged from $1=$ 'strongly disagree' to $4=$ 'strongly agree'. A mean score was calculated, with higher scores indicating better family functioning.

\section{Statistical analysis}

To test for differences in the distribution of covariates (e.g. demographics), parent psychosocial factors and child sleep outcomes (i.e. wake time, bedtime, sleep duration and sleep quality) by food security status, the $t$ test, $\chi^{2}$ test and Mann-Whitney $U$ test were used for normally distributed continuous variables, categorical variables and nonnormal continuous variables, respectively. Sleep quality was the only child sleep outcome that differed significantly by food security status, thus subsequent analyses focus on sleep quality only. The unadjusted association between food security status (household, adult and child level) and sleep quality was quantified using logistic regression models (Model 1). Separate multivariable logistic regression models were applied to quantify the adjusted relationship between food security status (household, adult and child) and sleep quality controlling for household income and covariates that showed a significant bivariate relationship $(P<0.05)$ with food security status (Model 2$)$.

Mediation analysis was conducted to test whether the adjusted relationship detected in Model 2 was mediated by parent psychosocial factors (Fig. 1). Specifically, the statistical modelling included three steps. Step 1 was to obtain the adjusted coefficients between food security status and the mediator by constructing linear regression models of continuous parent psychosocial factor scores as a function of food security status with the same adjustment included in Model 2 (depicted as path $a$ in Fig. 1). Step 2 was to construct multivariable logistic regression models between

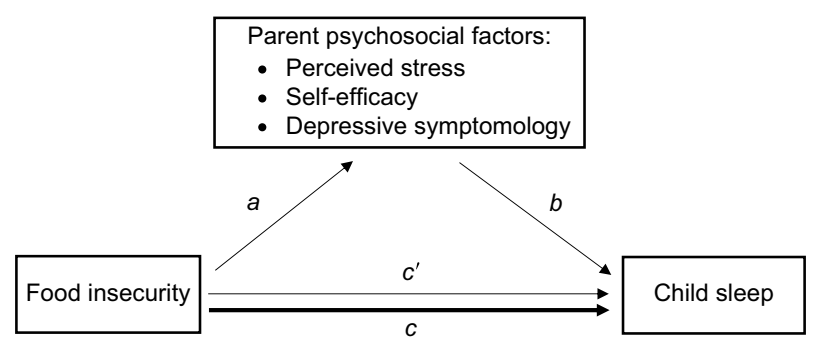

Fig. 1 Hypothesized mediation effect of parent psychosocial factors in the association between food insecurity and child sleep outcomes. a represents the adjusted association between food insecurity and the hypothesized mediator; $b$ represents the adjusted association between the hypothesized mediator and child sleep outcome, controlling for food insecurity; $c$ represents the total adjusted association between food insecurity and child sleep outcome (obtained from Model 2 in Table 2); and $c^{\prime}$ represents the adjusted association between food insecurity and child sleep outcome, controlling for the hypothesized mediator. The adjusted associations were controlled for number of people supported by household income, participation in assistance programmes, household income level, household chaos score and family functioning score 
food security status and the dichotomous child sleep outcomes, controlling for the mediator while adjusting for covariates included in Model 2. The second step generated two coefficients: the first representing the adjusted association between the mediator and sleep quality, controlling for food security status (depicted as path $b$ in Fig. 1); and the second representing the indirect association between food security status and sleep quality, controlling for the mediator (depicted as path $c^{\prime}$ in Fig. 1). Step 3 was to compare the total adjusted effect of food security status on sleep quality from Model 2 (depicted as path $c$ in Fig. 1) with the indirect effect obtained from step 2 (depicted as path $c^{\prime}$ in Fig. 1). All coefficients were standardized by multiplying by the standard deviation of the independent variable and dividing by the standard deviation of the dependent variable to achieve comparable coefficients obtained from linear and logistic models ${ }^{(41)}$. To quantify the magnitude of a potential mediation effect, two indicators were calculated using the standardized coefficients: (i) the percentage of the total effect that was mediated, calculated as $a \times b /\left(a \times b+c^{\prime}\right)$; and (ii) the ratio of the indirect effect to the direct effect, calculated as $a \times b / c^{\prime}$. The Sobel test was used to evaluate if the mediating path was statistically different from zero (the Sobel test statistic was calculated as the standardized coefficient $a \times b)^{(42)}$. All analyses were conducted using the statistical software package Stata SES 15.0 and imputation was performed using the statistical software package SAS version 9.4 .

\section{Results}

\section{Sample characteristics}

The majority of parents ( $95.8 \%$ female) and children were White (parents: 93.9\%, children: 86.9\%) non-Hispanic (parents: $97.9 \%$, children: $95.8 \%$ ). Additional sample characteristics, parent psychosocial factors and child sleep outcomes are presented in Table 1 by food security status. Of 362 parents included in the study, the prevalence of household, adult and child food insecurity was 37.3, 31.8 and $17.7 \%$, respectively. Household, adult and child food insecurity was associated with higher household chaos (all $P<0.005$ ) and lower family functioning (all $P<0.02$ ). Participation in SNAP was greater when participants reported household (food secure $v$. food insecure: $69 \cdot 1$ v. $85.0 \%, P=0.001)$ or adult (69.6 v. 86.7\%,P=0.0005) but not child (73.6 v. 82.3\%, $P=0.153)$ food insecurity. Participation in WIC was less common among households with food insecurity $(73.1 v .62 .7 \%, P=0.045)$ but did not differ by adult or child food security status. Parent perceived stress and depressive symptomology were significantly higher among families reporting food insecurity at all three levels (all $P \leq 0.0002$ ), while the parent self-efficacy score was significantly lower only in parents reporting child food insecurity $(P=0.043)$, but not household or adult food insecurity. Household chaos and family functioning were correlated with parent stress $\left(r_{\mathrm{CHAOS}}=0.51, r_{\mathrm{FAD}}=-0.46\right)$, depressive symptomology $\left(r_{\mathrm{CHAOS}}=0.41, r_{\mathrm{FAD}}=-0.37\right)$ and lower selfefficacy $\left(r_{\mathrm{CHAOS}}=-0.30, r_{\mathrm{FAD}}=0.31\right)$, all $P<0.01$ after Bonferroni correction.

\section{Child sleep outcomes by food security status}

In the overall sample, about half of parents reported that their child woke up prior to 07.00 hours (48.1\%) and went to bed between 20.00 and 21.00 hours (51.0\%). Mean (SD) sleep duration at night was $10 \cdot 7(0.9) \mathrm{h}$. Reported wake time, bedtime and sleep duration did not differ by food security status (all $P>0 \cdot 1$ ). The proportion of parents reporting maintaining a bedtime routine for more than $5 \mathrm{~d}$ /week was higher among food-secure (range: 77.2$78.4 \%$ ) compared with food-insecure (range: 67.2$70 \cdot 4 \%$ ) families, but these differences did not reach statistical significance (all $P>0 \cdot 08$ ). Compared with the food secure referent, the proportion of parents reporting poor child sleep quality (very poorly-well) was higher among parents reporting household (51.5 v. $63.7 \%, P=0.024)$, adult $(52.6 v \cdot 63.5 \%, P=0.053)$ and child $(52.7 v .71 .9 \%$, $P=0.005)$ food insecurity.

\section{Food security status and child sleep quality}

Table 2 presents odds ratios of poor child sleep quality by food security status before and after adjusting for participation in assistance programmes (e.g. SNAP), household chaos, family functioning and household income as covariates. In the unadjusted Model 1, household food insecurity was significantly associated with a 1.65-fold increased odds (95\% CI $1.07,2.55)$ of poor child sleep quality and child food insecurity was significantly associated with a $2 \cdot 30$-fold increased odds (95\% CI $1 \cdot 27,4 \cdot 14$ ) of poor child sleep quality (both $P<0.05$ ). However, food insecurity at the household level was not significantly associated with child sleep quality when covariates were included in Model 2. Child food insecurity was independently significantly associated with a $2 \cdot 27$-fold increased odds $(95 \%$ CI $1.12,4.59)$ of poor child sleep quality in the adjusted Model 2.

\section{Mediation tests of parent psychological factors}

The results from mediation tests of parent psychosocial factors are presented in Table 3. Compared with the adjusted odds of 2.27 (95\% CI 1.12, 4.59) in Model 2 (representing path $c$ depicted in Fig. 1), including parent psychosocial factors (perceived stress, self-efficacy and depressive symptomology $Z$-scores) in the model changed the odds for child food insecurity only slightly. The quantitative measure for the percentage of the effect mediated (all $\leq 4 \cdot 1 \%$ ) and the ratio between the indirect and direct effect were small (all $\leq 0 \cdot 05$ ). According to the Sobel test, none of the 


\section{NS Public Health Nutrition}

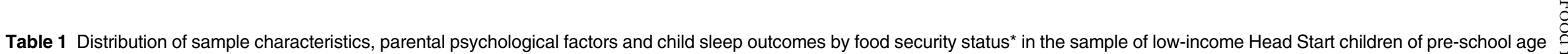
$(n 362)$ and their caregivers from four rural counties in central Pennsylvania, USA, May 2017-May 2018

\begin{tabular}{|c|c|c|c|c|c|c|c|c|c|c|c|c|c|c|c|c|}
\hline & \multirow[b]{3}{*}{$n$} & \multicolumn{4}{|c|}{ Household } & \multirow[b]{3}{*}{$P$ value } & \multicolumn{4}{|c|}{ Adult } & \multirow[b]{3}{*}{$P$ value } & \multicolumn{4}{|c|}{ Child } & \multirow[b]{3}{*}{$P$ value } \\
\hline & & \multicolumn{2}{|c|}{ Food secure } & \multicolumn{2}{|c|}{ Food insecure } & & \multicolumn{2}{|c|}{ Food secure } & \multicolumn{2}{|c|}{ Food insecure } & & \multicolumn{2}{|c|}{ Food secure } & \multicolumn{2}{|c|}{ Food insecure } & \\
\hline & & $\begin{array}{l}\%, \text { mean or } \\
\text { median }\end{array}$ & $\begin{array}{l}\text { SD or } \\
\text { IQR }\end{array}$ & $\begin{array}{l}\% \text {, mean or } \\
\text { median }\end{array}$ & $\begin{array}{l}\text { SD or } \\
\text { IQR }\end{array}$ & & $\begin{array}{l}\% \text {, mean or } \\
\text { median }\end{array}$ & $\begin{array}{l}\text { SD or } \\
\text { IQR }\end{array}$ & $\begin{array}{l}\% \text {, mean or } \\
\text { median }\end{array}$ & $\begin{array}{l}\text { SD or } \\
\text { IQR }\end{array}$ & & $\begin{array}{l}\% \text {, mean or } \\
\text { median }\end{array}$ & $\begin{array}{l}\text { SD or } \\
\text { IQR }\end{array}$ & $\begin{array}{l}\% \text {, mean or } \\
\text { median }\end{array}$ & $\begin{array}{l}\text { SD or } \\
\text { IQR }\end{array}$ & \\
\hline$\%$ & 362 & $62 \cdot 7$ & - & $37 \cdot 3$ & - & & $68 \cdot 2$ & - & $31 \cdot 8$ & - & & $82 \cdot 3$ & - & $17 \cdot 7$ & - & \\
\hline \multicolumn{17}{|c|}{ Child and parental characteristics } \\
\hline Child age† (years) & 357 & 4.4 & $0 \cdot 70$ & 4.5 & 0.76 & 0.514 & 4.4 & 0.71 & 4.5 & 0.74 & 0.382 & 4.4 & 0.70 & $4 \cdot 4$ & $0 \cdot 81$ & 0.443 \\
\hline $\begin{array}{l}\text { Child sex } \neq \\
\text { Male }\end{array}$ & 194 & 53.7 & - & & & 0.335 & & & & & 0.334 & & & & & 0.884 \\
\hline $\begin{array}{l}\text { Male } \\
\text { Female }\end{array}$ & & $\begin{array}{l}53 \cdot 7 \\
46 \cdot 3\end{array}$ & $\begin{array}{l}- \\
-\end{array}$ & $\begin{array}{l}46 \cdot 6 \\
53 \cdot 4\end{array}$ & $\begin{array}{l}- \\
-\end{array}$ & & $\begin{array}{l}53 \cdot 4 \\
46 \cdot 6\end{array}$ & $\begin{array}{l}- \\
-\end{array}$ & $\begin{array}{l}46 \cdot 0 \\
54 \cdot 0\end{array}$ & $\begin{array}{l}- \\
-\end{array}$ & & $\begin{array}{l}51 \cdot 3 \\
48 \cdot 7\end{array}$ & $\begin{array}{l}- \\
-\end{array}$ & $\begin{array}{l}50 \cdot 0 \\
50.0\end{array}$ & $\begin{array}{l}- \\
-\end{array}$ & \\
\hline Parent age§ (years) & 350 & $32 \cdot 3$ & $9 \cdot 81$ & 31.7 & 8.38 & 0.867 & $32 \cdot 2$ & 9.68 & 31.8 & 8.45 & 0.787 & 31.9 & 9.60 & $32 \cdot 6$ & $7 \cdot 78$ & 0.122 \\
\hline $\begin{array}{l}\text { Highest parent education } \\
\text { completed } \neq\end{array}$ & 358 & & & & & 0.385 & & & & & $0 \cdot 171$ & & & & & 0.412 \\
\hline$<$ High school & & $16 \cdot 5$ & - & $20 \cdot 1$ & - & & $16 \cdot 0$ & - & $21 \cdot 9$ & - & & $18 \cdot 6$ & - & $14 \cdot 3$ & - & \\
\hline$\geq$ High school & & 83.5 & - & 79.9 & - & & 84.0 & - & $78 \cdot 1$ & - & & $81 \cdot 4$ & - & $85 \cdot 7$ & - & \\
\hline Marital status $\ddagger$ & 358 & & & & & 0.345 & & & & & 0.341 & & & & & 0.736 \\
\hline $\begin{array}{l}\text { Single/divorced/ } \\
\text { separated/widowed }\end{array}$ & & $37 \cdot 5$ & - & $42 \cdot 5$ & - & & $37 \cdot 7$ & - & $43 \cdot 0$ & - & & $39 \cdot 0$ & - & $41 \cdot 3$ & - & \\
\hline Married or partnered & & $62 \cdot 5$ & - & 57.5 & - & & $62 \cdot 3$ & - & $57 \cdot 0$ & - & & $61 \cdot 0$ & - & 58.7 & - & \\
\hline Employment status $\ddagger$ & 356 & & & & & 0.760 & & & & & 0.647 & & & & & $0 \cdot 761$ \\
\hline Únemployed & & 52.5 & - & $54 \cdot 1$ & - & & $52 \cdot 3$ & - & 54.9 & - & & $52 \cdot 7$ & - & $54 \cdot 8$ & - & \\
\hline Employed & & 47.5 & - & $45 \cdot 9$ & - & & $47 \cdot 7$ & - & $45 \cdot 1$ & - & & $47 \cdot 3$ & - & $45 \cdot 2$ & - & \\
\hline \multicolumn{17}{|l|}{ Household characteristics } \\
\hline $\begin{array}{c}\text { Number of children }<18 \\
\text { years in household§ }\end{array}$ & 359 & $2 \cdot 5$ & 1.32 & $2 \cdot 4$ & $1 \cdot 10$ & 0.819 & $2 \cdot 4$ & $1 \cdot 29$ & $2 \cdot 5$ & $1 \cdot 12$ & 0.352 & $2 \cdot 5$ & $1 \cdot 26$ & $2 \cdot 3$ & $1 \cdot 12$ & 0.525 \\
\hline $\begin{array}{l}\text { Number of people } \\
\text { (supported by } \\
\text { income)§ }\end{array}$ & 333 & $4 \cdot 1$ & $1 \cdot 80$ & $3 \cdot 9$ & 1.54 & 0.405 & $4 \cdot 1$ & $1 \cdot 74$ & $4 \cdot 0$ & $1 \cdot 62$ & 0.928 & $4 \cdot 1$ & $1 \cdot 73$ & $3 \cdot 8$ & 1.58 & 0.289 \\
\hline $\begin{array}{l}\text { Annual household } \\
\text { income } \neq \text { (\$US) }\end{array}$ & 288 & & & & & 0.618 & & & & & 0.591 & & & & & 0.093 \\
\hline$<20000$ & & $46 \cdot 1$ & - & $49 \cdot 1$ & - & & $46 \cdot 1$ & - & 49.5 & - & - & $45 \cdot 0$ & - & $58 \cdot 0$ & - & \\
\hline$\geq 20000$ & & 53.9 & - & 50.9 & - & & 53.9 & - & $50 \cdot 5$ & - & - & $55 \cdot 0$ & - & $42 \cdot 0$ & - & \\
\hline \multicolumn{17}{|c|}{ Participation in assistance programme in the past 12 months $\ddagger$} \\
\hline SNAP/Food Stamps & 350 & 69.1 & - & 85.0 & - & 0.001 & $69 \cdot 6$ & - & $86 \cdot 7$ & - & 0.0005 & $73 \cdot 6$ & - & $82 \cdot 3$ & - & 0.153 \\
\hline WIC & 345 & $73 \cdot 1$ & - & $62 \cdot 7$ & - & 0.045 & $71 \cdot 1$ & - & $65 \cdot 1$ & - & 0.262 & $69 \cdot 6$ & - & $67 \cdot 8$ & - & 0.787 \\
\hline TANF & 306 & $8 \cdot 2$ & - & $10 \cdot 8$ & - & 0.447 & $8 \cdot 1$ & - & $11 \cdot 6$ & - & 0.323 & $7 \cdot 9$ & - & $15 \cdot 1$ & - & 0.099 \\
\hline $\begin{array}{c}\text { Any assistance } \\
\text { programme }\end{array}$ & 355 & $90 \cdot 1$ & - & $92 \cdot 5$ & - & 0.446 & $89 \cdot 7$ & - & $93 \cdot 8$ & - & 0.205 & $90 \cdot 1$ & - & $95 \cdot 2$ & - & 0.206 \\
\hline Region $\ddagger$ & 362 & & & & & 0.684 & & & & & 0.924 & & & & & 0.750 \\
\hline Bradford-Tioga & & $26 \cdot 0$ & - & $29 \cdot 6$ & - & & $26 \cdot 7$ & - & $28 \cdot 7$ & - & & $28 \cdot 5$ & - & $21 \cdot 9$ & - & \\
\hline Blair & & $34 \cdot 8$ & - & $29 \cdot 6$ & - & & $34 \cdot 0$ & - & $30 \cdot 4$ & - & & $32 \cdot 2$ & - & $35 \cdot 9$ & - & \\
\hline Huntingdon & & $21 \cdot 6$ & - & 24.4 & - & & $22 \cdot 3$ & - & 23.5 & - & & $22 \cdot 5$ & - & 23.4 & - & \\
\hline Snyder-Union-Mifflin & & $17 \cdot 6$ & - & $16 \cdot 3$ & - & & $17 \cdot 0$ & - & $17 \cdot 4$ & - & & $16 \cdot 8$ & - & $18 \cdot 8$ & - & \\
\hline
\end{tabular}


Table 1 Continued

\begin{tabular}{|c|c|c|c|c|c|c|c|c|c|c|c|c|c|c|c|c|}
\hline & \multirow[b]{3}{*}{$n$} & \multicolumn{4}{|c|}{ Household } & \multirow[b]{3}{*}{$P$ value } & \multicolumn{4}{|c|}{ Adult } & \multirow[b]{3}{*}{$P$ value } & \multicolumn{4}{|c|}{ Child } & \multirow[b]{3}{*}{$P$ value } \\
\hline & & \multicolumn{2}{|c|}{ Food secure } & \multicolumn{2}{|c|}{ Food insecure } & & \multicolumn{2}{|c|}{ Food secure } & \multicolumn{2}{|c|}{ Food insecure } & & \multicolumn{2}{|c|}{ Food secure } & \multicolumn{2}{|c|}{ Food insecure } & \\
\hline & & $\begin{array}{l}\% \text {, mean or } \\
\text { median }\end{array}$ & $\begin{array}{l}\text { SD or } \\
\text { IQR }\end{array}$ & $\begin{array}{l}\% \text {, mean or } \\
\text { median }\end{array}$ & $\begin{array}{l}\text { SD or } \\
\text { IQR }\end{array}$ & & $\begin{array}{l}\% \text {, mean or } \\
\text { median }\end{array}$ & $\begin{array}{l}\text { SD or } \\
\text { IQR }\end{array}$ & $\begin{array}{l}\% \text {, mean or } \\
\text { median }\end{array}$ & $\begin{array}{l}\text { SD or } \\
\text { IQR }\end{array}$ & & $\begin{array}{l}\% \text {, mean or } \\
\text { median }\end{array}$ & $\begin{array}{l}\text { SD or } \\
\text { IQR }\end{array}$ & $\begin{array}{l}\% \text {, mean or } \\
\text { median }\end{array}$ & $\begin{array}{l}\text { SD or } \\
\text { IQR }\end{array}$ & \\
\hline $\begin{array}{l}\text { Household chaos§ } \\
\text { (score) }\end{array}$ & 362 & $2 \cdot 3$ & 0.63 & $2 \cdot 6$ & 0.63 & $<0.0001$ & $2 \cdot 3$ & 0.64 & $2 \cdot 6$ & $0 \cdot 61$ & $<0.0001$ & $2 \cdot 3$ & 0.65 & $2 \cdot 6$ & 0.59 & 0.004 \\
\hline $\begin{array}{l}\text { Family functioning§ } \\
\text { (score) }\end{array}$ & 360 & $3 \cdot 6$ & 0.39 & $3 \cdot 4$ & 0.49 & 0.003 & $3 \cdot 6$ & 0.41 & 3.4 & 0.47 & 0.002 & $3 \cdot 6$ & 0.42 & $3 \cdot 4$ & 0.49 & 0.014 \\
\hline \multicolumn{17}{|c|}{ Parental psychological factors } \\
\hline $\begin{array}{l}\text { Perceived stress } \dagger \\
\quad(Z \text {-score })\end{array}$ & 361 & $-0 \cdot 2$ & 0.91 & 0.4 & 1.01 & $<0.0001$ & -0.2 & 0.92 & 0.5 & 1.00 & $<0.0001$ & -0.1 & 0.95 & 0.5 & 1.07 & $<0.0001$ \\
\hline Self-efficacy§ (Z-score) & 362 & 0.1 & 0.98 & $-0 \cdot 1$ & 1.04 & 0.124 & 0.0 & 0.98 & -0.1 & 1.04 & 0.102 & 0.0 & 1.00 & -0.2 & 0.97 & 0.043 \\
\hline $\begin{array}{l}\text { Depressive } \\
\text { symptomatology§ } \\
\text { (Z-score) }\end{array}$ & 362 & -0.2 & 0.87 & 0.4 & 1.09 & $<0.0001$ & -0.2 & $0 \cdot 86$ & 0.5 & $1 \cdot 10$ & $<0.0001$ & -0.1 & 0.96 & 0.4 & 1.09 & 0.0002 \\
\hline $\begin{array}{l}\text { Child sleep outcomes } \\
\text { Usual wake timeł }\end{array}$ & 362 & & & & & 0.429 & & & & & 0.710 & & & & & 0.961 \\
\hline$\leq 07.00$ hours & & $49 \cdot 8$ & - & $45 \cdot 2$ & - & & $48 \cdot 6$ & - & $47 \cdot 0$ & - & & $48 \cdot 3$ & - & $46 \cdot 9$ & - & \\
\hline $07.00-08.00$ hours & & 30.4 & - & $37 \cdot 0$ & - & & 31.6 & - & 35.7 & - & & $32 \cdot 6$ & - & 34.4 & - & \\
\hline$>08.00$ hours & & $19 \cdot 8$ & - & $17 \cdot 8$ & - & & $19 \cdot 8$ & - & $17 \cdot 4$ & - & & $19 \cdot 1$ & - & $18 \cdot 8$ & - & \\
\hline Usual bedtimeł & 361 & & & & & 0.180 & & & & & 0.196 & & & & & 0.672 \\
\hline$\leq 20.00$ hours & & 30.5 & - & $25 \cdot 9$ & - & & $30 \cdot 9$ & - & $24 \cdot 3$ & - & & $29 \cdot 6$ & - & $25 \cdot 0$ & - & \\
\hline $20.00-21.00$ hours & & $52 \cdot 2$ & - & 48.9 & - & & $51 \cdot 2$ & - & $50 \cdot 4$ & - & & $50 \cdot 8$ & - & $51 \cdot 6$ & - & \\
\hline$>21.00$ hours & & $17 \cdot 3$ & - & $25 \cdot 2$ & - & & $17 \cdot 9$ & - & $25 \cdot 2$ & - & & 19.5 & - & $23 \cdot 4$ & - & \\
\hline Sleep duration§ (h) & 361 & $10 \cdot 7$ & 0.95 & $10 \cdot 8$ & 0.94 & 0.860 & $10 \cdot 8$ & 0.94 & $10 \cdot 7$ & 0.96 & 0.945 & $10 \cdot 8$ & 0.95 & $10 \cdot 7$ & 0.93 & 0.564 \\
\hline $\begin{array}{l}\text { Bedtime routine } \\
\text { frequency per week } \ddagger\end{array}$ & 362 & & & & & 0.086 & & & & & 0.133 & & & & & 0.092 \\
\hline$<5$ nights & & $21 \cdot 6$ & - & $29 \cdot 6$ & - & & $22 \cdot 3$ & - & $29 \cdot 6$ & - & & $22 \cdot 8$ & - & $32 \cdot 8$ & - & \\
\hline 5-7 nights & & 78.4 & - & $70 \cdot 4$ & - & & $77 \cdot 7$ & - & $70 \cdot 4$ & - & & $77 \cdot 2$ & - & $67 \cdot 2$ & - & \\
\hline Reported sleep quality $\ddagger$ & 362 & & & & & 0.024 & & & & & 0.053 & & & & & 0.005 \\
\hline Very well & & 48.5 & - & $36 \cdot 3$ & - & & 47.4 & - & $36 \cdot 5$ & - & & $47 \cdot 3$ & - & $28 \cdot 1$ & - & \\
\hline Poor (very poorly-well) & & 51.5 & - & 63.7 & - & & $52 \cdot 6$ & - & 63.5 & - & & $52 \cdot 7$ & - & 71.9 & - & \\
\hline
\end{tabular}

IQR, interquartile range; SNAP, Supplemental Nutrition Assistance Program; WIC, Special Supplemental Nutrition Program for Women, Infants, and Children; TANF, Temporary Assistance for Needy Families. Statistically significant $P$ values are shown in bold.

*Values presented are percentages, or as means and standard deviations, or as medians and interquartile ranges.

†The $t$ test was conducted to determine differences between normally distributed continuous variables and binary food security status.

FThe $\chi^{2}$ test was conducted to determine differences between categorical variables and binary food security status.

$\S$ The Mann-Whitney $U$ test was used to determine differences between non-normal continuous variables and binary food security status. 
Table 2 Unadjusted and adjusted odds ratios (and $95 \%$ confidence intervals) of the associations between reported poorer child sleep quality and food security status at household, adult and child level in the sample of low-income Head Start children of pre-school age ( $n 362)$ and their caregivers from four rural counties in central Pennsylvania, USA, May 2017-May 2018

\begin{tabular}{|c|c|c|c|c|c|c|c|c|c|c|}
\hline & \multirow[b]{2}{*}{$n$} & \multicolumn{3}{|c|}{ Household } & \multicolumn{3}{|c|}{ Adult } & \multicolumn{3}{|c|}{ Child } \\
\hline & & OR & $95 \% \mathrm{Cl}$ & $P$ value & OR & $95 \% \mathrm{Cl}$ & $P$ value & OR & $95 \% \mathrm{Cl}$ & $P$ value \\
\hline \multirow{4}{*}{$\begin{array}{l}\text { Model 1: Unadjusted associations } \\
\text { Food insecure (ref. = food secure) } \\
\text { Model 2: Adjusted associations } \\
\text { Food insecure (ref. }=\text { food secure) }\end{array}$} & \multirow[t]{2}{*}{362} & & & & & & & & & \\
\hline & & 1.65 & $1.07,2.55$ & 0.02 & 1.56 & $0.99,2.46$ & 0.054 & $2 \cdot 30$ & $1.27,4.14$ & 0.01 \\
\hline & \multirow[t]{2}{*}{284} & & & & & & & & & \\
\hline & & 1.66 & $0.99,2.78$ & 0.06 & 1.56 & $0.91,2.68$ & 0.11 & $2 \cdot 27$ & $1.12,4.59$ & 0.02 \\
\hline
\end{tabular}

Ref., referent category.

Statistically significant values are shown in bold.

*Adjusted for participation in assistance programmes, household income level, household chaos score and family functioning score.

parent psychosocial factors significantly mediated the relationship between child food insecurity and child sleep quality (all Sobel test $P>0.5$ ).

\section{Discussion}

To our knowledge, the present study is the first to explore the association between food security status and sleep outcomes among young children of low-income families. We found a significant association between child, but not household or adult, food insecurity and sleep quality in children of pre-school age after adjusting for a number of potential confounders and covariates, including the number of people supported by household income, participation in assistance programmes (e.g. SNAP), household chaos, family functioning and household income. In contrast to our hypothesis, the association between child food insecurity and child sleep quality was not mediated by the parent psychosocial factors of interest.

As hypothesized, food-insecure children were about two times more likely to have poor sleep quality compared with food-secure children. Several mechanisms may explain the association in the present study. One potential pathway may be related to children's emotional and behavioural health. Although food insecurity experience is rarely expressed in young children, food insecurity as a direct stressor was found to increase risk of several behavioural problems in pre-school children ${ }^{(43,44)}$, which correlated with shorter sleep duration ${ }^{(45)}$ and other sleep problems ${ }^{(46)}$. The biological pathway explaining stress as a potential mediator is hypothesized to be related to an impaired immune system and disrupted hypothalamicpituitary-adrenal axis activity ${ }^{(47)}$, which are both involved in modulating sleep ${ }^{(48)}$. Although food insecurity is not a measure of hunger, children directly impacted by food insecurity may be more likely to experience hunger, which could affect sleep quality particularly if a child goes to bed hungry.

The present study observed a lack of association of household and adult food insecurity with child sleep outcomes. The lack of association may be interpreted in light of the growing literature exploring the relationship between low socio-economic status (SES) and the home environment on child sleep outcomes. Several studies have reported that children aged 1-17 years from lowSES families have more sleep problems such as short sleep duration and poor sleep quality compared with their higher-income counterparts ${ }^{(49,50)}$. Recent evidence from longitudinal studies further supports the association of low SES and material deprivation, defined through the quality of the house and neighbourhood where a child lives, with sleep later in childhood and adolescence ${ }^{(47,51)}$. Among pre-school children, Crabtree et al. ${ }^{(52)}$ found higher impaired sleep scores and problematic bedtime behaviours in children from lower-SES groups than in those from higher-SES groups in a large sample of children aged 2-7 years ( $n$ 3371). Emerging evidence supporting the association between SES and child sleep outcomes may explain, in part, why we did not see an association between adult and household food insecurity and child sleep quality after adjusting for household income and home environment factors (i.e. chaos and family functioning).

Consistent with previous research, parent psychosocial factors, including depressive symptoms ${ }^{(53,54)}$ and perceived stress $^{(55,56)}$, were associated with higher risk of child food insecurity (Table 1) and poorer child sleep quality (see online supplementary material, Supplemental Table S1). Building on previous research examining a potential relationship between parenting and food insecurity ${ }^{(31,57)}$, we found a positive relationship between parenting selfefficacy and both food insecurity and poorer child sleep quality. However, these parent psychosocial factors did not explain the observed association between child food insecurity and poor sleep quality. Given that factors within the home environment, such as higher household chaos and lower family functioning, were associated with increased parent stress, depressive symptoms and lower self-efficacy in the current study sample, adjusting for these home environment factors may have attenuated a true mediation effect. We also cannot rule out the possibility 
that other unmeasured parental psychosocial constructs may have had a mediating effect that we could not examine in our study.

Findings from the present study need to be interpreted acknowledging a number of limitations. First, the present study was cross-sectional in nature; thus, the temporality of food insecurity and child sleep outcomes and causality could not be determined. Second, sleep outcomes (duration, routine and quality) were assessed via parent report, which is subject to recall bias. For example, errors in reported bedtime and wake time, especially among children who do not follow a regular sleep routine, could have caused some random variability in the calculated sleep duration, which, at least partially, led to the insignificant findings when exploring the relationship between food insecurity and sleep duration. For the sleep quality measure, it is likely that parents have subjectively underestimated their children's sleep problems, such as night wakings ${ }^{(58)}$. Previous research has shown that number of night wakings is one of the major factors by which parents judge the quality of their child's sleep ${ }^{(59)}$. Thus, objective sleep measures such as actigraphy may provide more accurate measurements and should be considered in future studies with young children ${ }^{(60)}$. Third, there were missing data in household income that reduced the analytic sample. However, sensitivity analyses without adjusting for household income and adjusting using imputed household income values showed robust results (see online supplementary material, Supplemental Tables S2 and S3). Finally, we cannot eliminate the risk of non-response bias given the nature of the study design and the moderate survey response rate. Therefore, the external validity of our findings may be limited to certain low-income families. Although less than desirable, the response rate was similar to that in another study utilizing survey data collected in a similar manner from a rural Head Start population in Colorado (i.e. $28.5 \%)^{(61)}$. Future studies are warranted to test whether or not our study findings can be generalized beyond Head Start children in rural central Pennsylvania.

\section{Conclusion}

In conclusion, results from the present study suggest that child food insecurity may be associated with suboptimal sleep quality among children of pre-school age and this association may not be mediated by parent psychosocial factors. Sleep problems during early stages of life can have detrimental effects on the cognitive, socio-emotional and physical health of children throughout the life course. Food insecurity, particularly at the child level, is a potential modifiable risk factor for reducing sleep-related health disparities in early childhood. Evidence from our study highlights the importance of focusing on children's experience of food insecurity and its potential impact on their sleep quality and related health outcomes. Future studies are 
needed to explore the potential mechanisms underlying the associations between child food insecurity and adverse sleep outcomes.

\section{Acknowledgements}

Acknowledgements: The authors acknowledge Michele E. Marini at the Center for Childhood Obesity Research, Penn State University, for managing and preparing the data sets for data analysis. Financial support: This research was supported by US Department of Agriculture's Supplemental Nutrition Assistance Program (SNAP) through the PA Department of Human Services (DHS). The research reported in this publication was supported by the National Center for Advancing Translational Sciences of the National Institutes of Health (grant numbers TL1 TR002016 and UL1 TR002014). The content is solely the responsibility of the authors and does not necessarily represent the official views of the NIH. Conflict of interest: The authors have no conflicts of interest relevant to this article. Authorship: J.S.S. designed the study. M.N. conceived the research question, conducted data analysis and prepared the first draft of the manuscript. S.G.E., L.J., K.L. and J.S.S. helped with literature review, data interpretation, manuscript writing and revising. All authors read and approved the final manuscript. Ethics of buman subject participation: This study was conducted according to the guidelines laid down in the Declaration of Helsinki and all procedures involving human subjects were approved by the Office for Research Protections at The Pennsylvania State University, University Park, PA, USA. Implied informed consent was obtained from all subjects.

\section{Supplementary material}

To view supplementary material for this article, please visit https://doi.org/10.1017/S136898001900332X

\section{References}

1. Touchette É, Petit D, Paquet J et al. (2005) Factors associated with fragmented sleep at night across early childhood. Arch Pediatr Adolesc Med 159, 242-249.

2. Smaldone A, Honig JC \& Byrne MW (2007) Sleepless in America: inadequate sleep and relationships to health and well-being of our nation's children. Pediatrics 119, Suppl. 1, S29-S37.

3. Sadeh A, Gruber R \& Raviv A (2002) Sleep, neurobehavioral functioning, and behavior problems in school-age children. Child Dev 73, 405-417.

4. Dewald JF, Meijer AM, Oort FJ et al. (2010) The influence of sleep quality, sleep duration and sleepiness on school performance in children and adolescents: a meta-analytic review. Sleep Med Rev 14, 179-189.
5. Cappuccio FP, Taggart FM, Kandala N-B et al. (2008) Metaanalysis of short sleep duration and obesity in children and adults. Sleep 31, 619-626.

6. Patel SR \& Hu FB (2008) Short sleep duration and weight gain: a systematic review. Obesity (Silver Spring) 16, 643-653.

7. Chen X, Beydoun MA \& Wang Y (2008) Is sleep duration associated with childhood obesity? A systematic review and meta-analysis. Obesity (Silver Spring) 16, 265-274.

8. Kobayashi K, Yorifuji T, Yamakawa M et al. (2015) Poor toddler-age sleep schedules predict school-age behavioral disorders in a longitudinal survey. Brain Dev 37, 572-578.

9. Sivertsen B, Harvey AG, Reichborn-Kjennerud T et al. (2015) Later emotional and behavioral problems associated with sleep problems in toddlers: a longitudinal study. JAMA Pediatr 169, 575-582.

10. Armstrong JM, Ruttle PL, Klein MH et al. (2014) Associations of child insomnia, sleep movement, and their persistence with mental health symptoms in childhood and adolescence. Sleep 37, 901-909.

11. Coleman-Jensen A, Rabbitt MP, Gregory CA et al. (2016) Household Food Security in the United States in 2015. Washington, DC: US Department of Agriculture, Economic Research Service.

12. Ding M, Keiley MK, Garza KB et al. (2014) Food insecurity is associated with poor sleep outcomes among US adults. J Nutr 145, 615-621.

13. Liu Y, Njai RS, Greenlund KJ et al. (2014) Relationships between housing and food insecurity, frequent mental distress, and insufficient sleep among adults in 12 US States, 2009. Prev Chronic Dis 11, E37.

14. Jordan ML, Perez-Escamilla R, Desai MM et al. (2016) Household food insecurity and sleep patterns among Mexican adults: results from ENSANUT-2012. J Immigr Minor Health 18, 1093-1103.

15. Whinnery J, Jackson N, Rattanaumpawan P et al. (2014) Short and long sleep duration associated with race/ethnicity, sociodemographics, and socioeconomic position. Sleep 37, 601-611.

16. US Department of Agriculture (2018) Food Security Status of US Households with Children in 2017. https://www.ers.usda. gov/topics/food-nutrition-assistance/food-security-in-the-us/ key-statistics-graphics.aspx ${ }^{\#}$ children (accessed September 2018).

17. Rocha NP, Milagres LC, Novaes JFd et al. (2016) Association between food and nutrition insecurity with cardiometabolic risk factors in childhood and adolescence: a systematic review. Rev Paul Pediatr 34, 225-233.

18. Eisenmann JC, Gundersen C, Lohman BJ et al. (2011) Is food insecurity related to overweight and obesity in children and adolescents? A summary of studies, 1995-2009. Obes Rev 12, e73-e83.

19. Shankar P, Chung R \& Frank DA (2017) Association of food insecurity with children's behavioral, emotional, and academic outcomes: a systematic review. J Dev Behav Pediatr 38, $135-150$.

20. Maynard M, Andrade L, Packull-McCormick S et al. (2018) Food insecurity and mental health among females in highincome countries. Int J Environ Res Public Health 15, 1424.

21. Lovejoy MC, Graczyk PA, O'Hare E et al. (2000) Maternal depression and parenting behavior: a meta-analytic review. Clin Psychol Rev 20, 561-592.

22. Mindell JA, Li AM, Sadeh A et al. (2015) Bedtime routines for young children: a dose-dependent association with sleep outcomes. Sleep 38, 717-722.

23. Sadeh A, Mindell JA, Luedtke K et al. (2009) Sleep and sleep ecology in the first 3 years: a web-based study. J Sleep Res $\mathbf{1 8}$, 60-73. 
24. Sadeh A (2004) A brief screening questionnaire for infant sleep problems: validation and findings for an Internet sample. Pediatrics 113, e570-e 577.

25. Mindell JA, Sadeh A, Kwon R et al. (2013) Cross-cultural differences in the sleep of preschool children. Sleep Med 14, 1283-1289.

26. Kushnir J \& Sadeh A (2011) Sleep of preschool children with night-time fears. Sleep Med 12, 870-874.

27. Gundersen C \& Ziliak JP (2014) Childhood food insecurity in the US: trends, causes, and policy options. Future Child Fall 2014, 1-19.

28. Cohen S, Kamarck T \& Mermelstein R (1983) A global measure of perceived stress. J Health Soc Behav 24, 385-396.

29. Lee E-H (2012) Review of the psychometric evidence of the perceived stress scale. Asian Nurs Res 6, 121-127.

30. Gilmore L \& Cuskelly M (2009) Factor structure of the parenting sense of competence scale using a normative sample. Child Care Health Dev 35, 48-55.

31. Heerman WJ, Taylor JL, Wallston KA et al. (2017) Parenting self-efficacy, parent depression, and healthy childhood behaviors in a low-income minority population: a crosssectional analysis. Matern Child Health J 21, 1156-1165.

32. Andresen EM, Malmgren JA, Carter WB et al. (1994) Screening for depression in well older adults: evaluation of a short form of the CES-D. Am J Prev Med 10, 77-84.

33. Appelhans BM, Fitzpatrick SL, Li H et al. (2014) The home environment and childhood obesity in low-income households: indirect effects via sleep duration and screen time. BMC Public Health 14, 1160.

34. Boles RE, Halbower AC, Daniels S et al. (2017) Family chaos and child functioning in relation to sleep problems among children at risk for obesity. Behav Sleep Med 15, 114-128.

35. Matheny AP Jr, Wachs TD, Ludwig JL et al. (1995) Bringing order out of chaos: psychometric characteristics of the confusion, hubbub, and order scale. J Appl Dev Psychol 16, 429-444

36. Corapci F (2008) The role of child temperament on Head Start preschoolers' social competence in the context of cumulative risk. J Appl Dev Psychol 29, 1-16.

37. Dumas JE, Nissley J, Nordstrom A et al. (2005) Home chaos: sociodemographic, parenting, interactional, and child correlates. J Clin Child Adolesc Psychol 34, 93-104.

38. Hart SA, Petrill SA, Deater-Deckard K et al. (2007) SES and CHAOS as environmental mediators of cognitive ability: a longitudinal genetic analysis. Intelligence 35, 233-242.

39. Epstein NB, Baldwin LM \& Bishop DS (1983) The McMaster family assessment device. J Marital Fam Ther 9, 171-180.

40. Miller IW, Epstein NB, Bishop DS et al. (1985) The McMaster family assessment device: reliability and validity. $J$ Marital Fam Ther 11, 345-356.

41. MacKinnon DP \& Dwyer JH (1993) Estimating mediated effects in prevention studies. Eval Rev 17, 144-158.

42. Preacher KJ \& Hayes AF (2004) SPSS and SAS procedures for estimating indirect effects in simple mediation models. Behav Res Methods Instrum Comput 36, 717-731.

43. Melchior M, Chastang J-F, Falissard B et al. (2012) Food insecurity and children's mental health: a prospective birth cohort study. PLoS One 7, e52615.

44. Weinreb L, Wehler C, Perloff J et al. (2002) Hunger: its impact on children's health and mental health. Pediatrics 110, e41.
45. Lavigne JV, Arend R, Rosenbaum D et al. (1999) Sleep and behavior problems among preschoolers. J Dev Behav Pediatr 20, 164-169.

46. Hiscock H, Canterford L, Ukoumunne OC et al. (2007) Adverse associations of sleep problems in Australian preschoolers: national population study. Pediatrics 119, 86-93.

47. Barazzetta M \& Ghislandi S (2016) Family income and material deprivation: do they matter for sleep quality and quantity in early life? Evidence from a longitudinal study. Sleep 40, zsw066.

48. Buckley TM \& Schatzberg AF (2005) On the interactions of the hypothalamic-pituitary-adrenal (HPA) axis and sleep: normal HPA axis activity and circadian rhythm, exemplary sleep disorders. J Clin Endocrinol Metab 90, 3106-3114.

49. El-Sheikh M, Kelly RJ, Buckhalt JA et al. (2010) Children's sleep and adjustment over time: the role of socioeconomic context. Child Dev 81, 870-883.

50. El-Sheikh M, Bagley EJ, Keiley M et al. (2013) Economic adversity and children's sleep problems: multiple indicators and moderation of effects. Health Psychol 32, 849-859.

51. Doane LD, Breitenstein RS, Beekman C et al. (2019) Early life socioeconomic disparities in children's sleep: the mediating role of the current home environment. $J$ Youth Adolesc $\mathbf{4 8}$, 56-70.

52. Crabtree VM, Korhonen JB, Montgomery-Downs HE et al. (2005) Cultural influences on the bedtime behaviors of young children. Sleep Med 6, 319-324.

53. Whitaker RC, Phillips SM \& Orzol SM (2006) Food insecurity and the risks of depression and anxiety in mothers and behavior problems in their preschool-aged children. Pediatrics 118, e859-e868.

54. de Jong DM, Cremone A, Kurdziel LB et al. (2016) Maternal depressive symptoms and household income in relation to sleep in early childhood. J Pediatr Psychol 41, 961-970.

55. Laraia BA, Siega-Riz AM, Gundersen C et al. (2006) Psychosocial factors and socioeconomic indicators are associated with household food insecurity among pregnant women. J Nutr 136, 177-182.

56. Caldwell BA \& Redeker NS (2015) Maternal stress and psychological status and sleep in minority preschool children. Public Health Nurs 32, 101-111.

57. Bronte-Tinkew J, Zaslow M, Capps R et al. (2007) Food insecurity works through depression, parenting, and infant feeding to influence overweight and health in toddlers. J Nutr 137, 2160-2165.

58. Sadeh A (1996) Evaluating night wakings in sleep-disturbed infants: a methodological study of parental reports and actigraphy. Sleep 19, 757-762.

59. Palmstierna P, Sepa A \& Ludvigsson J (2008) Parent perceptions of child sleep: a study of 10000 Swedish children. Acta Paediatr 97, 1631-1639.

60. Meltzer LJ, Biggs S, Reynolds A et al. (2012) The children's report of sleep patterns-sleepiness scale: a self-report measure for school-aged children. Sleep Med 13, 385-389.

61. McCloskey M, Johnson SL, Benz C et al. (2018) Parent perceptions of mobile device use among preschool-aged children in rural Head Start centers. J Nutr Educ Behav 50, 83-89.e1. 